\title{
A FRAMEWORK FOR SEMANTIC DRIVEN ELECTRONIC EXAMINATION SYSTEM FOR SUBJECTIVE QUESTIONS
}

\author{
H. A Zubairu1,", I. O. Oyefolahan ${ }^{2}$, S. O. Etuk ${ }^{3}$ and F. J. Babakano ${ }^{4}$

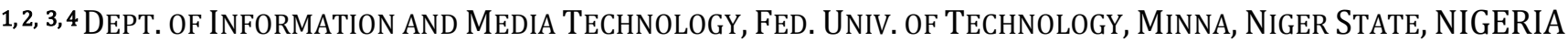 \\ E-mail addresses: ${ }^{1}$ abu.zubairu@futminna.edu.ng, ${ }^{2}$ o.ishaq@futminna.edu.ng, \\ 3abiolastella@futminna.edu.ng, ${ }^{4}$ faiza.bkano@futminna.edu.ng
}

\begin{abstract}
In this paper, a theoretical framework that incorporates a semantic dictionary (WordNet) into the current eexamination system to support subjective question is proposed. The WordNet serves as a medium of enhancing the current e-examination system in which machines can process, interpret, and evaluate information for enhance knowledge retrieval. The approach adopted is hypothesized to solve the semantic mismatch issues in subjective eexamination. An algorithm that explores the synset structure of WordNet database and which gives meaning of any term based on the available synonyms is proposed. The framework is implemented using Java programming language and a prototype of the proposed system is tested and compared with the existing system. Results show that words that are synonymous to any given correct answer are equally recognize as correct option. Hence, the e-examination system reliability, performance and assessment of depth of knowledge of students have been greatly increased.
\end{abstract}

Keywords: E-examination, subjective question, WordNet, semantic

\section{INTRODUCTION}

Examination is an important process for the evaluation of teaching and learning activities for the completion of teaching and learning where level of information from the instructor are evaluated to assess either the teaching or the learning process along all levels [9].

Since the inception of education, there have been several techniques for examination process [14]. The evolution and technology advancement has led to the emergence and popularity of electronic examination (eexamination) system. E-examination is the utilization of Information and Communication Technology (ICT) equipment and devices for an assessment or evaluation of knowledge [13]. It is an assessment approach meditated by electronic technology and personal computer is a vital component of the system [22]. It allows examiner(s) to author and plan examinations through the computer system where responses are electronically recorded and assessed [13]. The aim is to make examination or assessment process faster and efficient. The existing electronic examination systems are majorly in the form of Multiple Choice Questions (MCQs). This form of online examination question could only determine the overview knowledge of a subject [14].

E-examination is no doubt replacing the conventional paper and pencil based examination system across the globe. The acceptance of computer-based assessment in learning by teachers and students in Nigerian schools is becoming widespread [2]. Although, the electronic examination is highly applauded due to its effectiveness and efficiency, but its limitation lies in the depth of knowledge that can be assessed [21]. The need for the assessment of an in-depth knowledge of subject necessitate the need for electronic examination system that support subjective questions like fill in the blanks, match the pair, true or false type questions, one word answer, definition type question, one sentence answer and sometimes descriptive type of questions [21].

Although, subjective e-examination systems have been proposed, the current system need to be examined carefully. The e-examination system for subjective questions depends strongly on syntax match in evaluating the correct answer. To determine an in depth knowledge similar to that of traditional examination system, the system should take cognizance of the semantics in evaluating the answer. Semantic 
evaluation is necessary because there are different viewpoints and assumptions regarding what are essentially the same concept. Therefore, evaluation and comparing of user's inputs with the designated correct answer is not possible without at least some semantic knowledge [5].

To address this issue, this paper developed a model to integrate WordNet (a semantic dictionary), with the current e-examination system to improve the evaluation and performance of the existing eexamination system. This approach will make it possible for the system to perform the same evaluation of syntax match, to the designated answers, even if they are syntactically dissimilar.

\subsection{Semantic Heterogeneity}

Semantic heterogeneity occurs when the same real world entity, modelled by two or more people, does not have the same model or representation [9]. Due to the fact that these models are developed independently, they may have different terminologies, or interpretations, presenting a hindrance for machine to reason semantically. These conflicts result from the use of different names for the same entity in the same domain.

Ontolog have been suggested as a cornerstone to solve the problem of semantic heterogeneity. The basis is that ontology offers a shared common understanding of the application domain. It provide the meaning of terms and their relationships that facilitate communication by providing precise notions that can be use to compose messages about the domain [20].

In this direction, this paper utilizes WordNet ontology to serve as a controlled vocabulary and semantic similarity comparison mechanism between concepts. If semantic relation like synonyms could be incorporate in the existing e-examination database, semantically related terms will be accepted by the system. By introducing this mechanism that can identify semantically similar terms, challenges posed by the use of different terms to define the same concept may no longer be an issue in the case of subjective question on the e-examination platform.

\subsection{WordNet as Semantic Dictionary}

WordNet [19] is an online lexical resource for English language that classify synonymous terms into synsets, each expressing a distinct concept. The WordNet database contains information on semantic relatedness for words, including relations such as synonym (same meaning), antonym (opposite in meanings), hyponym (hierarchical relationship), and meronym (part-of relationship). WordNet labels the semantic relations among the words and provides explicit meaning of terms [10].

WordNet, a semantic dictionary is a good resource for mapping concepts. The user's input is extended with the synonyms in WordNet belonging to the synset of each term contained in the designated answer(s). Though, WordNet contains different relations, this paper only exploits the synonyms part of the WordNet. WordNet is used to generate semantically meaningful option by deriving the synonyms related to the available correct option(s).

The rest of the paper is organized as follows; section 2 is the background of study and problem statement, section 3 discussed the methodology, the synonym clustering algorithm and system architecture, section 4 is for result and discussion and performance evaluation, conclusion and future work is in section 5 . Section 6 is for references.

\section{BACKGROUND OF STUDY}

Assessment is an integral part of learning whose measurement is challenging [12]. Assessing the learning of a student is interesting and important affair that requires diligence. Online assessment is gaining popularity and acceptance by many examination bodies and higher institutions of learning. However, most of the existing online assessment supports only objective type of questioning in form of multiple choice questions. The scope of this type of question is limited to perception and retention without demanding the student to be effective in his/her thinking capacity [12]. Therefore, education experts suggest on evaluating the students by adopting a hybrid approach that incorporates both the objective as well as subjective types of questioning methodologies [6].

Unlike the objective type of e-examination system, it is more difficult to design a system with capability to supports subjective type questions. Such systems requires concept of ontology or semantic knowledge for context aware provision of learning material and machine understandably with a goal to improve learner's performance.

Guang-Zou, et al [8] proposed an ontology based grid for educational applications. The project called Onto Edu is architecture for concept reusability with ontology, device and user adaptability with ubiquitous computing and automatic composition. It is divided into four parts: User adaptation, Service composition, Education ontology, Semantic Educational service. Though, the research is quite innovative, however, its major focus is geared towards adaptability or 
personalization. The architecture of this proposed framework lacks the capability to resolve the semantic heterogeneity required for effective implementation of subjective e-examination implementation.

Adekoya, Akinwale and Sofoluwe [1] described a conceptual framework for an ontology-based examination system and the ontology required for such examination was based on the methontology development technique proposed by [7]. The paper presented the concepts using a tree-like class hierarchy that indicate relationship between the super-class concept and the sub-class concepts. Protégé_4.0_alpha which is based on the web ontology language descriptive logic (OWL-DL) was used as a developing tool. FaCT++ reasoner was used to check for the consistency, computation and the validity of the inferred ontology. Examination ontology was developed to provide a knowledge base for the semantic examination grid. The design of the ontology was done with a view to permit integration of additional concepts in the future and at different levels of content granularity. The concept of this paper is a move towards machine process-able data; however, the methontology technique adopted may not address general application. Instead of proposing a framework from the scratch as proposed in [1], with scalabilities and integration issues, this paper suggest an approach that involves modification to the existing e-examination platforms.

Samir and Hosam [16] used semantic web to support the creation and evaluation of open questions in elearning courses. The objective of the research was to use Semantic Web and Wireless technologies to design and implement the assessment system that allows students; to take web based tutorials, quizzes, free exercises, and exams. This paper claims to have included semantic in their work, this cannot be verify from the research. There is no report of data used in the evaluation. Instead, the main focus of this paper is to measure the effects of web based and Mobile-based assessments on the achievement levels, performance and perceptions of the students in internet-enabled courses

Aslihan [4] developed an online exam system, in which students can take examination from computers and other mobile environments. It is a platform that allows users with an electronic environment to easily and quickly take examination from any location. The platform designed in this paper is only for multiple choice questions which can only examine overview knowledge, there is no provision on the system for handling subjective or filling the blank type of question.
Shinde and Chokhadrex [18] proposed a novel approach of an e-examination system that supports both objective and subjective questions. The system is no doubt an enhancement on the objectives eexamination platform. Nevertheless, the system is syntax sensitive. The user is expected to type the exact keyword without cognizance of the synonyms. Thus, semantic heterogeneity is an issue in this research. This drawback will be address in this paper.

Sheeba [17] proposed an algorithm for the automatic evaluation of single sentence descriptive answer. The paper presents an approach to check the degree of learning of the learner, by evaluating user's descriptive examination answer sheets. The descriptive answers are represented in the form of graph and compared with standard answer. This paper presents only a theoretical approach to realise a semantic based subjective e-examination. However, there is no implementation carried out to verify the feasibility of the approach. Technically, this paper is an advancement that implements framework proposed in [17].

Namrata, et al [11] developed a mobile based examination system. The authors maintained that the existing online examination is basically in the form of MCQs and argue that it can only examine overview knowledge of a particular subject. Therefore, a subjective electronic examination system for an in depth knowledge of subject area was proposed in the paper. Nevertheless, the paper focuses on making the examination more convenient by converting the conventional examination to an electronic based. Semantic heterogeneity that could arise due to the existence of synonyms in natural language was not addressed in [11]. Thus, this research may face issues of semantic heterogeneity, if it becomes operational.

\subsection{Problem Statement}

The electronic examination system for subjective question is sensitive to syntax in matching and evaluation of the correct answer. However, natural language is very rich in expression; therefore the same meaning can be conveyed in different forms using different set of word [17]. In the same way, answers evaluation for the same question is highly subjective and different instructors may have different views. This capability is still lacking in the current subjective e-examination system.

A typical screen shot of the subjective question is shown in Figure 1. A synonym of a term could have been used by a candidate, but because it does not 
match (the given answer syntactically), the candidate will be wrongly scored.

For example, a candidate that provides an answer "laptop" to the question in Figure 1 should have been score correctly, since it is synonymous to the answer "Computer", but such candidate would be wrongly scored because his or her response is not syntactically same with the answer. The current system does not take cognizance of the fact that, different terms or words may have the same or a similar meaning

Matching candidate's responses that are semantically related to the designated correct answer(s) might improve students' performance. But manually listing all the synonyms or semantically related in the system is not feasible. Nevertheless, if semantic relation like synonyms could be incorporated in the system, then semantically related terms could equally be scored as the correct answer by the system. Incorporating of synonymous term into the current system would be a difficult task without a semantic dictionary. By using WordNet, which has the capability of identifying semantically similar terms, challenges posed by the use of different terms to define the same concept would be handled. For instance, how would the computer know that 'personal computer', PC, and 'computer system' implies the same thing?

\section{METHODOLOGY}

In the existing e-examination platform, a user might input a term or phrase as the correct answer of choice and which is unknown, the system would check the WordNet database for a set of matches and determine if the designated answer is among the synset of the user choice. This is a complex task; since the system is syntax based. Therefore, there is need to design a concept that will be useful for this approach.

Define $S=\left\{s_{1}, s_{2}, s_{3},------s_{n}\right\}$, to be the set of synonyms retrieved from the WordNet, where $s_{k} \varepsilon S\left(s_{k}\right.$ is element of $S$ ), where $\mathrm{k}=1,2,3 \mathrm{n}$. It is a mapping from word phrases $(S)$ to sense phrases $\left(s_{k}\right)$, that is $S \rightarrow S_{k}$. In particular, the mapping $S \rightarrow s_{k}$, connotes the following: $S$ denotes a meaning of the (word) phrase $s_{\mathrm{k}}$. For example: Television $\rightarrow$ "TV", "Telly", "television", "telecast". This denotes the fact that the meaning of the (television) is expressed in the senses of "TV", "Telly", "television", and "telecast". The words "television" and "tv" are semantically equivalent. For mapping of semantically equivalent term, Porter stemmer is utilized to reduce each word to its base form.

From the problem definition, user may likely not enter exact term that matches the answer syntactically, however, the user(s) should be able to use or enter term or phrase that are semantically similar to the right answer. Thus, a means was device to define how terms can be semantically or conceptually related. Therefore, terms or concepts relatedness is define as $\mathrm{S}=\left\{\mathbf{s}_{1}, \mathrm{~s}_{2}, \mathrm{~s}_{3}\right.$, --------sn $\}$. For instance, Ssyn(computer) $=\{$ Laptop, Pc, Monitor\}. The set of synonyms are retrieved from WordNet [19] database. The retrieved synonyms are rank based on semantic similarity compare with the designated correct term(s). This was implemented using WordNet::Similarity [15], which contains algorithms for measuring semantic similarity. This aspect is shown in Figure 2 and the synonyms clustering algorithm in section 3.1 .

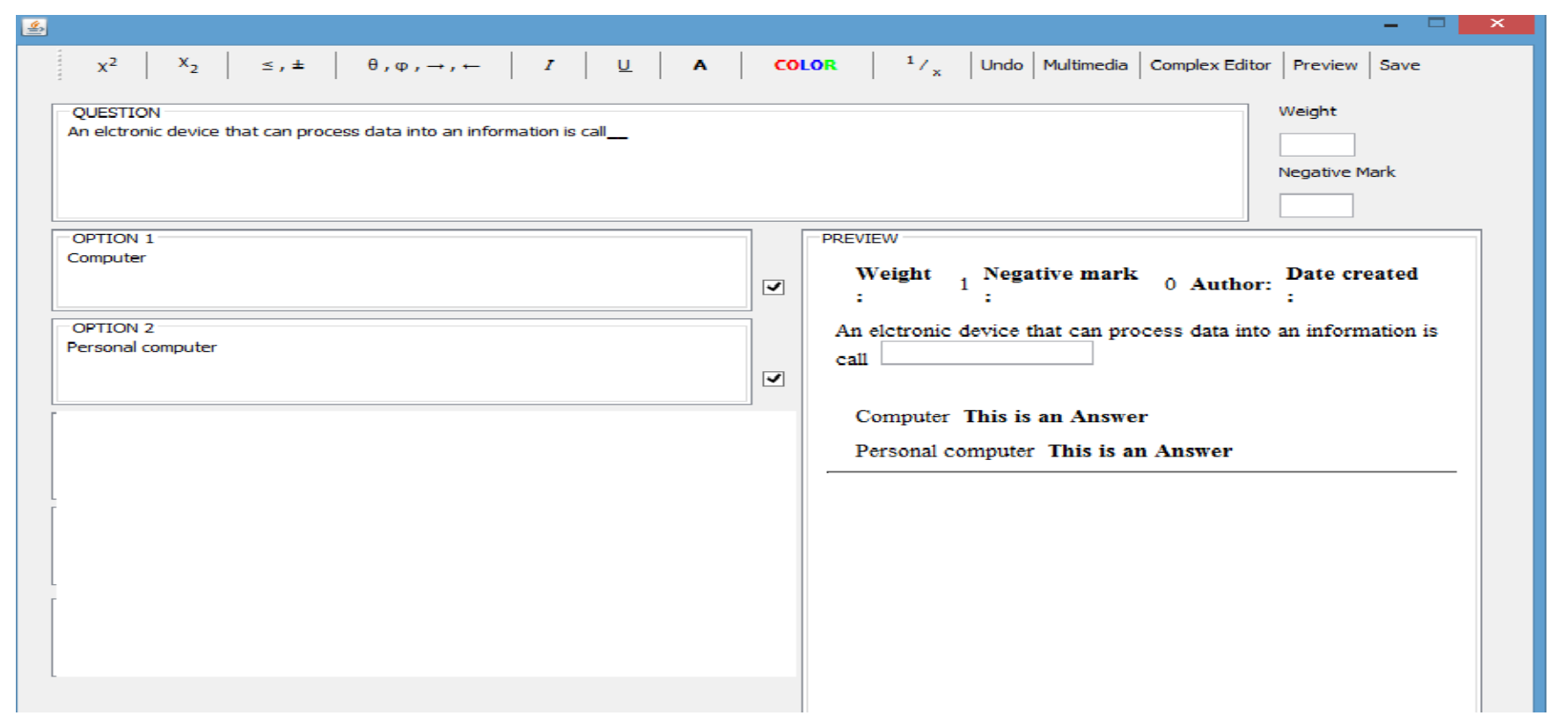

Figure 1: Screen shot of subjective question 


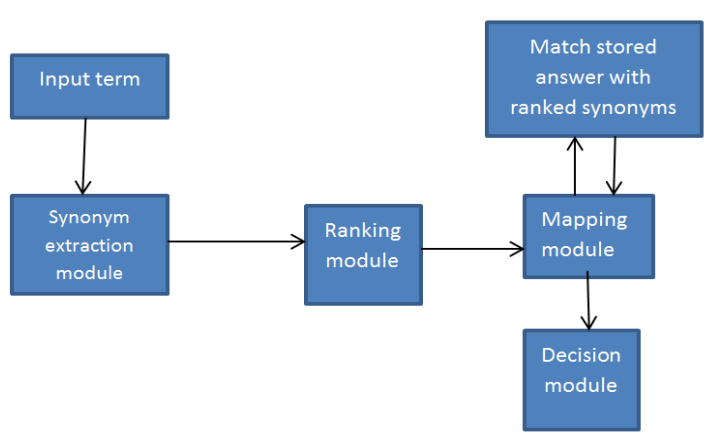

Figure 2: Synonyms extraction framework
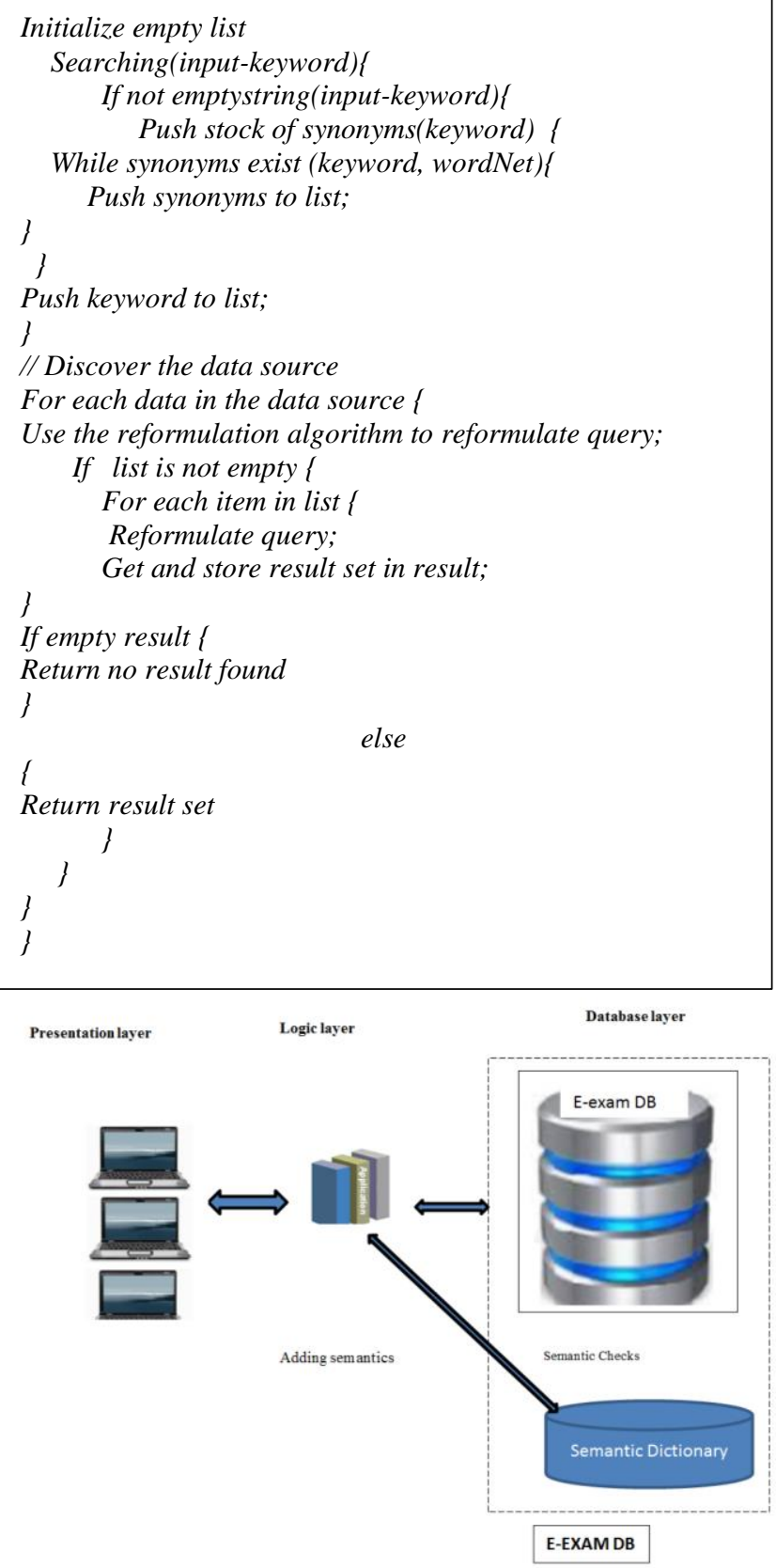

Figure 3: The system architecture of semantic -driven electronic examination system
Internally, WordNet uses Java WordNet search application interface (JAWS), a Java API to WordNet, to access the database. Thus, this application needs Java enable system for installation and functioning. The complete system architecture is shown in Figure 5.

\subsection{Synonym Clustering Algorithm}

This section shows an algorithm that explores the synonyms structure of WordNet database

\subsection{System Architecture}

The proposed electronic system is based on the existing system architecture that comprises the presentation tier, the logic tier and the database tier. However, unlike the existing structure, the proposed system database layer is a combination of the electronic examination database and semantic dictionary database intended to contain the likely terms instead of the existing system that is solely based on syntax. This system will provide an enhanced examination platform that handle semantic based responses from the users.

The semantic dictionary provides a common vocabulary of examination terminologies with different levels of formality, the meaning of the terms and the relationships between them. The semantic dictionary is expected to provide common and shared terms. It serves as knowledge bases which can be accessed if the system does not found a match from the keyword term. Figure 3 ; depict the architecture for a semantic driven eexamination system.

As shown in Figure 3, the first layer is the presentation layer, which is saddle with the responsibility of establishing connection between the client and the entire system. Client application software is embedded at the presentation layer. This layer is used for the presentation to user or accepts input from the user. The client can interact with the system through the form on web pages to the web server.

The second layer is the logic layer. This is the logic application that manages the whole system in term of client requests by a specific treatment process, and produces a series of Hypertext Mark-up Language (HTML) code at runtime, then sends the result to the client web browser. If the client requests data access, web server and database server will need to complete the task altogether [10]. The layer acts as an interface between application layer and data source layer. It is an intermediary layer that helps to make communication faster between application and data source layer.

The third layer is the database layer, which is mainly responsible for receiving requests from a web server, 
and then returns the results to the application layer, so as to realize data management. The database components of the system are design with Java Server Pages (JSP) and Structural Query Language (SQL) Server database technology. Unlike, the existing e-examination architecture, the proposed semantic driven eexamination system has an incorporated semantic dictionary database.

The database server contains two databases; the examination database and the semantic database. The semantic database plays a vital role of extending the meaning of terms and concepts.

The system is designed to check for semantic similarity of the user supply term in the semantic dictionary, in case a syntactic match is not found. Figure 4, is a flow chart that illustrates the operation of the proposed system.

\section{RESULTS AND DISCUSSION}

The evaluation of the framework was performed by implementing a database where terms and its corresponding possible synonyms are stored in different relational tables. The main interface to the system is shown in Figure 5.

Start

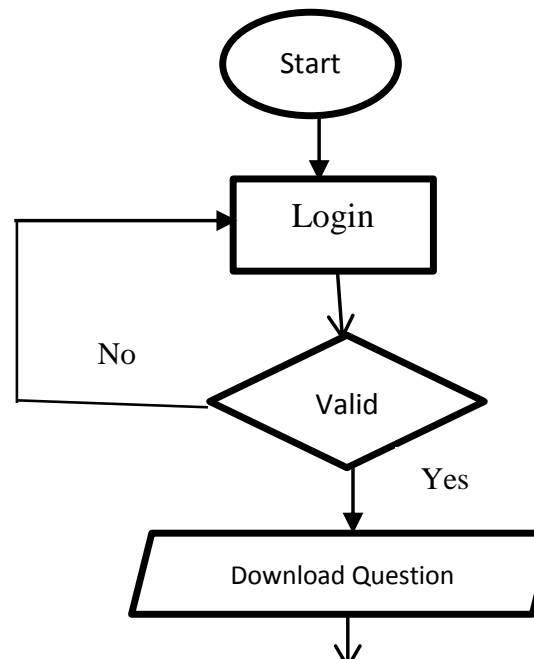

xtract

question

Record zero

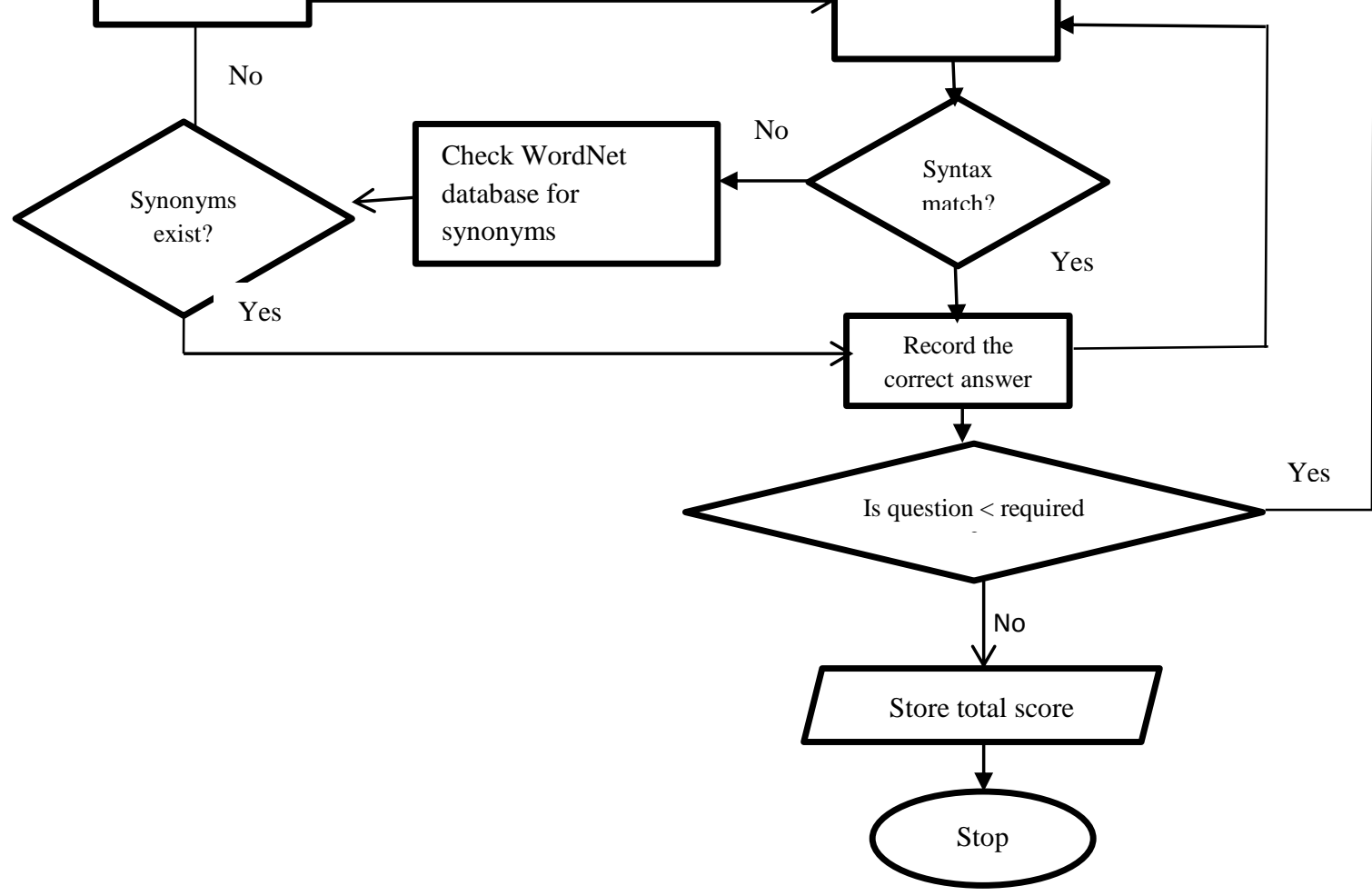

Figure 4: Flow chart of the proposed semantic driven e-exam system 


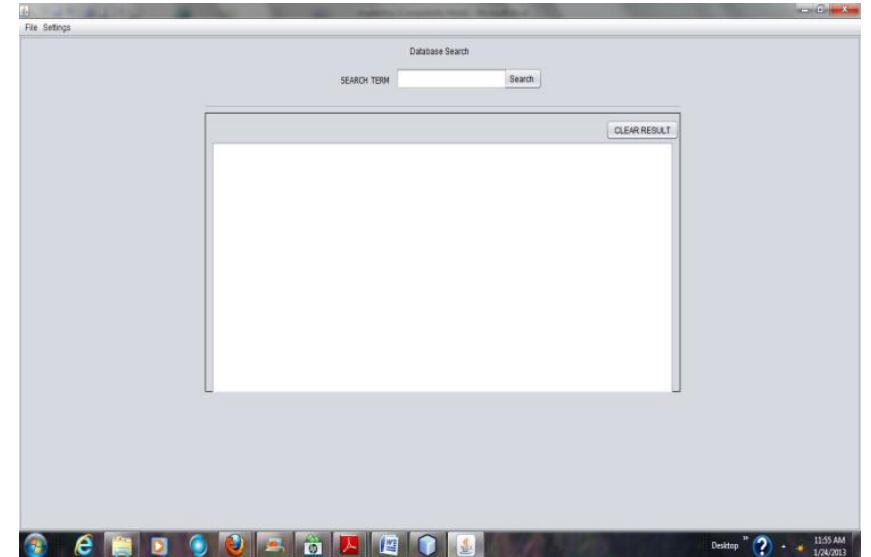

\section{Figure 5: The main GUI}

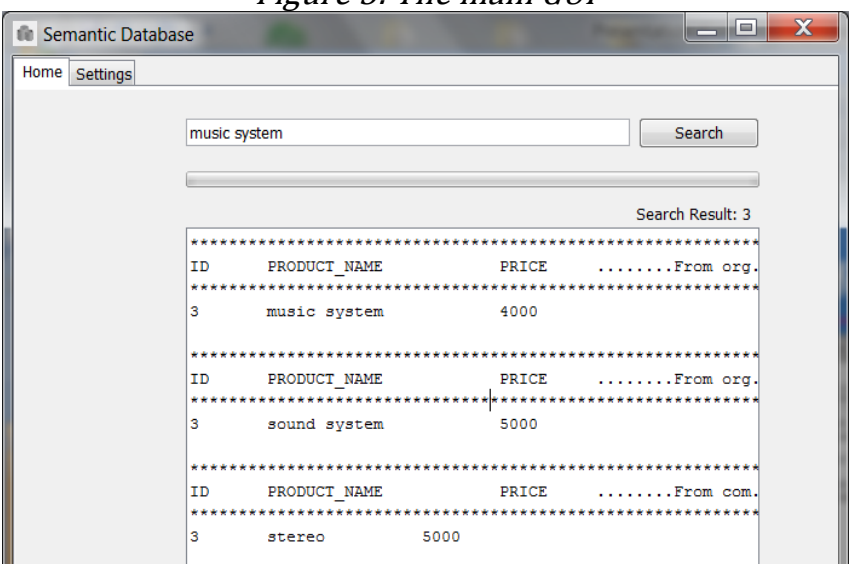

Figure 7: The system interface showing displaying result in the result panel for music system

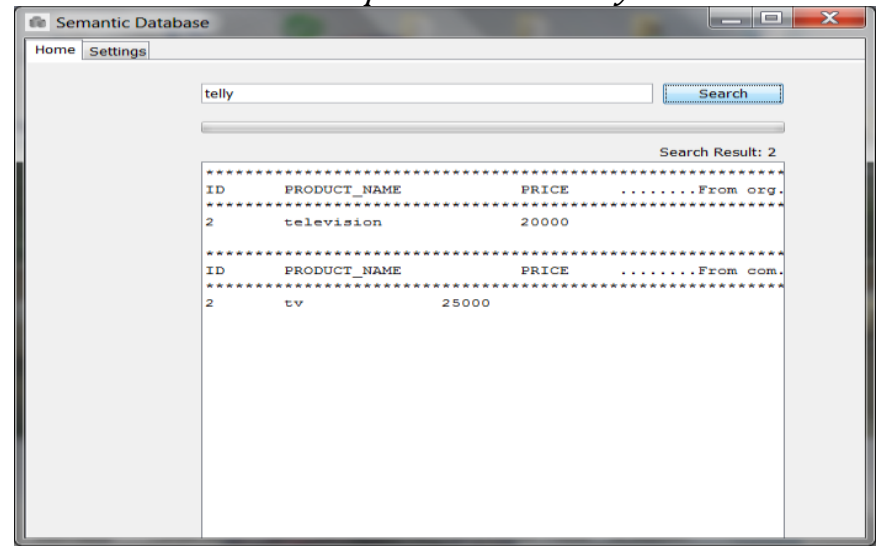

Figure 9: The system interface showing displaying result in the result panel for telly

The Figure 5, shows the main Graphical User Interface (GUI). Java swing is used to design the GUI. It has two menus; the first menu is file menu. It has just one submenu-the exit menu. In addition, the GUI has a textbox that allows the user to search for term and a result panel to view the result.

The first evaluation was done without the semantic dictionary, and it was observed that if user specifies a term which is not present in the database, it result in no hit, even though, the intended concepts or entity are available. Figure 6, is the screen shot of no hit result.

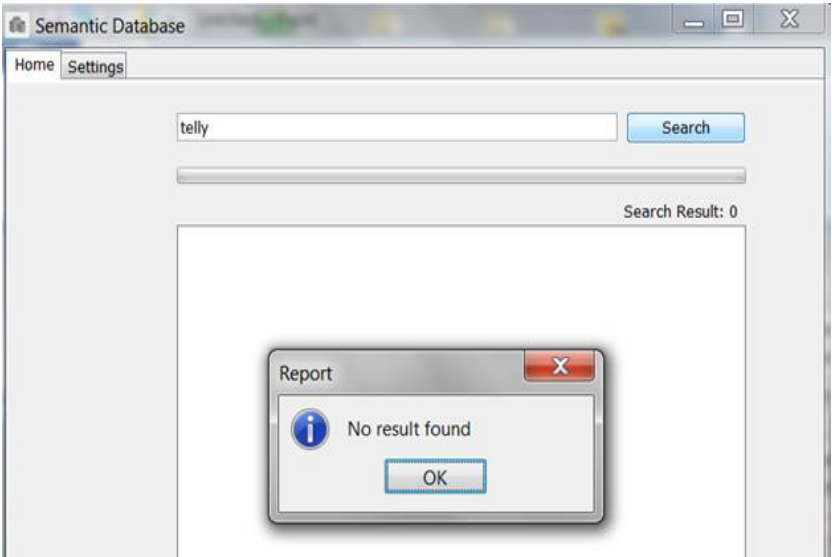

Figure 6: Screen shot of failed hit

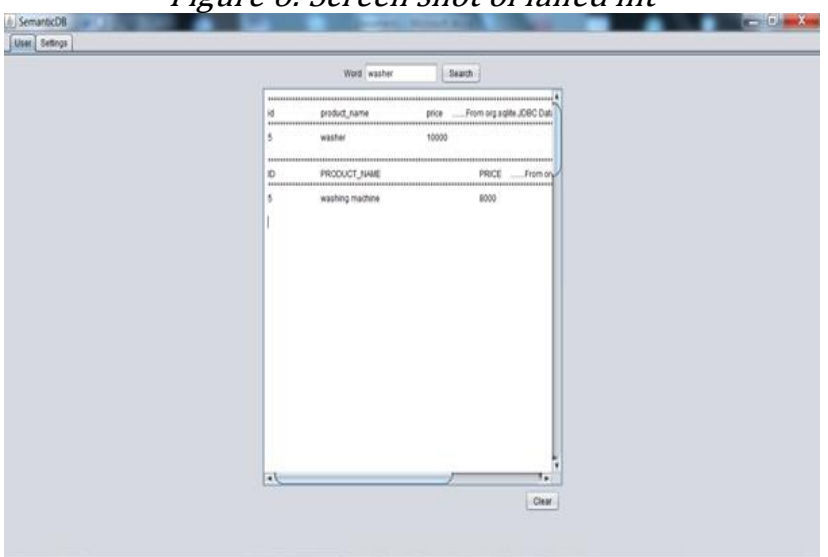

Figure 8: The system interface showing displaying result in the result panel for washer

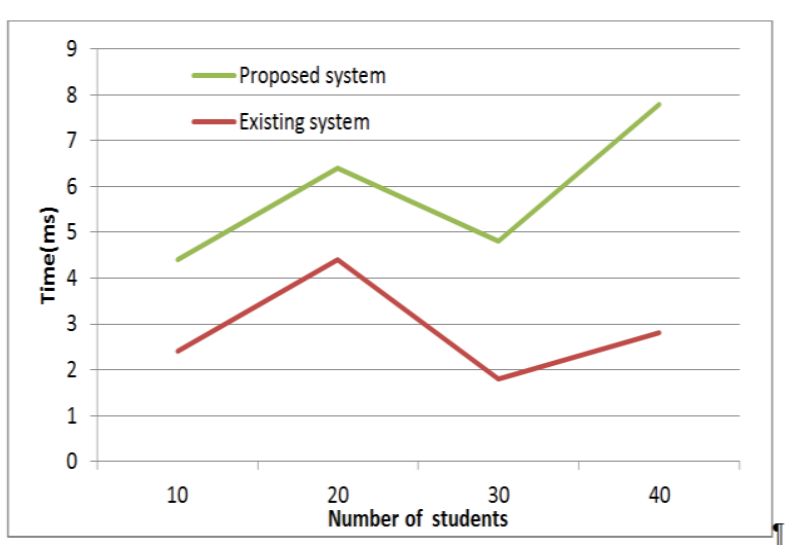

Figure 10: Time Response of the proposed and existing system

The subsequent evaluation was done with the semantic dictionary fully incorporated and integrated into the database. A query was executed on the database and obtains the concepts or entities that match with the user's request. Semantic dictionary not only discovers the concept or entities that syntactically match with that of the request, it also retrieves concepts or entities that are synonymous when the exact match is not found.

On giving a search term, semantically similar results are retrieved. For instance, a query for a music system, 
washing machine and television, output stereo, washer and telly respectively, as shown in Figure 7, 8 and 9, respectively.

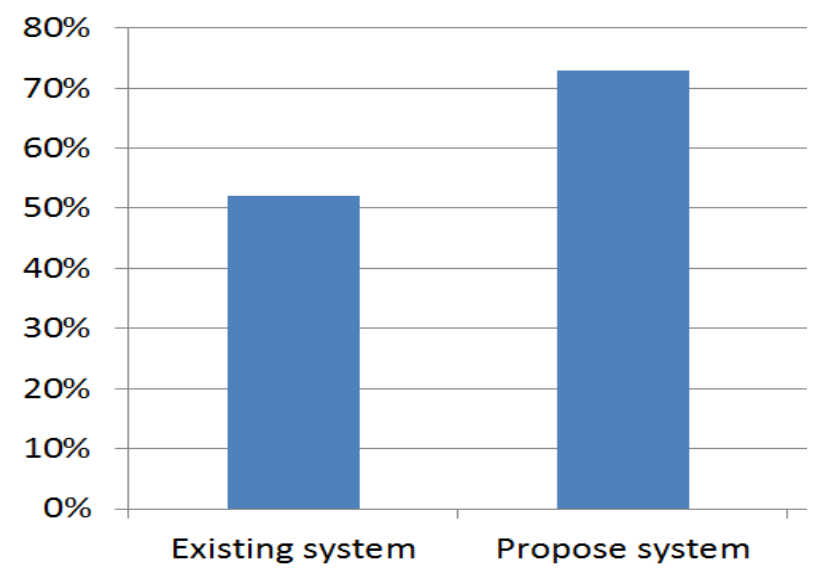

Figure 11: Test Results of the proposed and existing system

Table 1: Comparison of the proposed system with existing system

\begin{tabular}{lll}
\hline S/n & Existing E-exam system & Proposed E-exam system \\
\hline 1 & Easy to implement & $\begin{array}{l}\text { Require more effort to } \\
\text { design and implement } \\
\text { Support both objective and } \\
\text { objective questions }\end{array}$ \\
3 & $\begin{array}{l}\text { Mostly useful for } \\
\text { objective questions }\end{array}$ & $\begin{array}{l}\text { Semantic based matching } \\
\text { There is semantic } \\
\text { dictionary for synonyms } \\
\text { There is artificial } \\
\text { intelligence behaviour }\end{array}$ \\
\hline
\end{tabular}

As observed from the Figures 7, 8 and 9, even though, some instances where the term specified by the user is not available in the database, the system retrieves more semantically matching results. The system use the semantic dictionary to infers that music system, washer and television are all synonymous to stereo, washing machine and television respectively. The above results show an enhancement in the ability of retrieving term not only on exact syntax, but also on the existence of semantic relationships between the label terms. Thus, inclusion of sematic in search query improves recall as reported in [3]. The inclusion of semantic dictionary into the current e-examination system, enhance the capability of existing system to effectively support subjective questions, where different terms could have the same meaning or interpretation.

\subsection{Performance Evaluations}

The evaluation of the proposed framework with respect to the existing system was performed in term of response time and performance score. The same sets of questions are executed on the existing system and the proposed framework.

The response time of the proposed system was discovered to be higher than the existing system. The graph is shown in Figure 10. The inclusion of semantically related terms might have results in the increase of the response time. This result is in tandem with the assertion in [3] that semantic inclusion in query processing is not to speed up query processing but to provide users with additional meaningful answers.

In addition, the system was evaluated in term of students' score. Users were subject to take the same test using the proposed system and the existing system. Forty users were selected in performing the evaluation. The students take the test using the existing system and also the proposed system. The percentage score was discovered to be higher from the result of the proposed system. This improvement can be explained in term of high recall as a result of semantic inclusion. This result is corroborated by [15]. The graph is shown in Figure 11. The difference is clearly an indication that the proposed framework would lead to an increase in students' performance. The comparison of the proposed system with the existing system is show in Table 1.

\section{CONCLUSION AND FUTURE WORK}

This paper presents a framework that incorporates a semantic dictionary (WordNet) into an electronic examination platform to address the issue of syntactic dissimilarity in subjective e-examination. The prototype was successfully tested using some terms and its synonyms. The performance of the system was adjudged satisfactory by the users. By incorporating sematic dictionary into system, it is observe that performance in term of pass mark was better compare to the existing system. However, the response time was found to be higher in the proposed system. In future this system could be improved by addressing the issue of descriptive questions.

\section{REFERENCES}

[1] Adekoya A. F., Akinwale A. T. and Sofoluwe, A. A Conceptual Framework for an Ontology-Based Examination System, International Journal of Advanced Computer Science and Applications, Vol. 2, No. 5, pp. $36-42,2011$.

[2] Agbonifo, 0. C. An Online Model for Assessing Students' Understanding with stepwise solving of calculus questions, Nigerian Journal of Technology, vol. 33. no. 1, pp. 86 - 96, Jan. 2014. 
[3] Amshakala, K .and Nedunchezhian, R. WordNet Ontology Based Query Reformulation and Optimization using Disjunctive Clause Elimination, International Journal of Database Management Systems, Vol.3, No.4, pp. 53 - 64, Nov. 2011

[4] Aslihan T. "Development of an internet-based exam system for mobile environments and evaluation of its usuability" Melvana International Journal of Education, Vol. 3, No. 4, pp. 57 - 74, 2013.

[5] Budanitsky, A. and Hirst, G.Evaluating wordnetbased measures of lexical semantic relatedness. Comput. Linguist., 32(1): 13-47. 2006.

[6] Desimone, L, Werner M. and Harris M., Human Resource Development. 3rd Edition, USA, Harcourt College Publishers, 2002

[7] Fernández-López, M., Gómez-Pérez, A. and Juristo, N. METHONTOLOGY: From Ontological Art Towards Ontological Engineering. In: "AAAI-97 Spring Symposium Series", 24-26 March 1997, Stanford University, EEUU

[8] Guang-zuo, C., Fei, C., Hu, C., Li, S. "OntoEdu: Ontology based Education Grid System for elearning", GCCCE2004 International conference, Hong Kong. 2004.

[9] Juan Z., Wen-Yu, C. Li X. “Ontologybased teaching resources and courseware development system design", Computer Engineering and Design. Vol. 5, No. 6, pp. 1449 - 1457, 2007.

[10] Lenzerini, M. Data integration: a theoretical perspective. Proceedings of the twenty-first ACM SIGMOD-SIGACT-SIGART Symposium on Principles of database systems, pages 233-246, 2002.

[11] Namrata, P., Kalpesh, D.,Shivam Thakur, A. S. Virtual Subjective Examination on Tablets, International Journal of Computer Science and Information Technologies, Vol. 6 (5) , 4689-4691, 2015.

[12] Naveed A., Imran N., and Kashif U, Online Examination System and Assessment of Subjective Expression, International Conference on Education Technology and Computer, 2009.
[13] Omotehinwa T. O. and Durojaye D. (2013). Computer-Based Test: Security and Result Integrity. International Journal of Computer and Information Technology Vol. 2, No. 2, pp. 329 339, March 2013.

[14] Parekh, N., Dusane, K., Thakur, S. and Sonawane, A. Virtual Subjective Examination on Tablets, International Journal of Computer Science and Information Technologies, Vol. 6 (5), 4689-4691, 2015.

[15] Pedersen, T., Banerjee, S., and Patwardhan, S. Maximize semantic relatedness to perform word sense disambiguation, University of Minnesota Supercomputing Institute for Research, 2005.

[16] Samir A. E. and Hosam, F. E. Towards Development of Web-based Assessment System Based on Semantic Web Technology, Conference ICL2010 September 15 -17, 2010 Hasselt, Belgium. 2010.

[17] Sheeba P. An Approach to Evaluate Subjective Questions for Online Examination System, International Journal of Innovative Research in Computer and Communication Engineering, Vol. 2, Issue 11, pp. 6410 - 6413, November 2014

[18] Shinde, A. A. and Chokhandre, S. A Novel Approach With Subjective Assessment For EExamination, International Journal of Engineering Research and Applications, Vol. 3, Issue 4, pp. 3236, Jul-Aug. 2013.

[19] Wordnet Documentation : http://wordnet. princeton.edu. (assessed on Non. 10, 2016)

[20] Xiao L., Randy, C. An Ontology-based Mapping Repository for Dynamic and Customized Data Integration. (CISE Technical Report), University of Florida, United State, 2009.

[21] Vimal P.P., and Kumbharana, CK. Analysis Of Different Examination Patterns Having Question Answer Formulation, Evaluation Techniques And Comparison Of MCQ Type With One Word Answer For Automated Online Examination, International Journal of Scientific and Research Publications, Volume 6, Issue 3, March 2016.

[22], Iloanusi, 0. N. E-learning: Its implementation in higher institutions in Nigeria, Nigerian Journal of Technology, Vol. 26, No. 1, pp. 47 - 58, March 2007. 